\title{
New clues to the nature of immunoglobulin G4-related disease: a retrospective Japanese multicenter study of baseline clinical features of 334 cases
}

Kazunori Yamada ${ }^{1,2+}$, Motohisa Yamamoto ${ }^{3 \dagger}$, Takako Saeki ${ }^{4}$, Ichiro Mizushima', Shoko Matsui ${ }^{5}$, Yuhei Fujisawa ${ }^{1}$, Satoshi Hara ${ }^{1}$, Hiroki Takahashi ${ }^{3}$, Hideki Nomura ${ }^{5,6}$, Shigeyuki Kawa ${ }^{7}$ and Mitsuhiro Kawano ${ }^{*^{*}}$ (D)

\begin{abstract}
Background: The aim was to further characterize immunoglobulin G4-related disease (IgG4-RD) by a large-scale multicenter study of its clinical and laboratory features conducted by multidisciplinary physicians of IgG4-RD in Japan.

Methods: Various specialists retrospectively evaluated IgG4-RD patients diagnosed between 1996 and 2015 in five hospitals by analyzing their baseline clinical features, laboratory, imaging, and pathological test findings, and treatment.

Results: Of the 334 patients listed, 205 were male and median age at diagnosis was 65 years. The mean number of organs involved was 3.2 at diagnosis. The most frequently affected organs were the salivary glands, followed by the lacrimal glands, lymph nodes, pancreas, retroperitoneum/periaorta, kidneys, and lungs. The mean serum level of $\operatorname{lgG} 4$ was $755 \mathrm{mg} / \mathrm{dl}$, and more than $95 \%$ of patients had elevated serum lgG4 levels. The median serum level of C-reactive protein (CRP) was $0.1 \mathrm{mg} / \mathrm{dl}$ and the level was less than $1 \mathrm{mg} / \mathrm{dl}$ in $90 \%$ of patients. A total of $34.7 \%$ of patients had low serum levels of C3. Serum levels of C3 and non-lgG4 IgG, calculated as the total $\lg G$ minus $\lg G 4$, showed an inverse correlation in patients with kidney lesions, while serum IgG4 levels were not correlated with serum C3 levels. Corticosteroid was administered in 78.0\% of patients, and was effective in all.

Conclusions: The serum CRP level is generally low and the serum lgG4 level is elevated in most Japanese lgG4-RD patients, in contrast to western patients. These original findings suggest that these two parameters in IgG4-RD differ in some interesting ways from those hitherto reported in western populations. Additional studies, especially international comparative ones, are needed to elucidate the extent and significance of these differences between populations. Attention will also have to be paid to whether the existence of such differences requires consideration when devising international classification criteria.
\end{abstract}

Keywords: IgG4-related disease, Clinical features, Hypocomplementemia

\footnotetext{
* Correspondence: sk33166@gmail.com

${ }^{\dagger}$ Equal contributors

'Division of Rheumatology, Kanazawa University Hospital, 13-1, Takara-machi,

Kanazawa, Ishikawa 920-8640, Japan

Full list of author information is available at the end of the article
}

\section{Ciomed Central}

(c) The Author(s). 2017 Open Access This article is distributed under the terms of the Creative Commons Attribution 4.0 International License (http://creativecommons.org/licenses/by/4.0/), which permits unrestricted use, distribution, and reproduction in any medium, provided you give appropriate credit to the original author(s) and the source, provide a link to the Creative Commons license, and indicate if changes were made. The Creative Commons Public Domain Dedication waiver (http://creativecommons.org/publicdomain/zero/1.0/) applies to the data made available in this article, unless otherwise stated. 


\section{Background}

Immunoglobulin G4 (IgG4)-related disease (IgG4-RD), a widely recognized systemic inflammatory disorder [1-3], affects a broad range of organs with main features of elevated serum IgG4 levels, copious infiltration of IgG4positive cells into the affected organs, and a characteristic fibrosis called storiform fibrosis [2, 4]. IgG4-RD affects a broad range of organs such as the pancreas, lacrimal glands, salivary glands, kidneys, lungs, retroperitoneum and/or periaorta, skin, and lymph nodes [3, 5-8]. Owing to its extremely diverse clinical picture, individual clinicians encounter a clinically distinct patient population depending on his/her specialty and geographic location, making it difficult to avoid institutional bias in a single-center experience. Recently, several larger cohort studies have sought to characterize the clinical characteristics of IgG4-RD of over 100 patients [9-11]. Although these reports documented the baseline clinical features of IgG4-RD, differences were seen in the frequency of the affected organs and some laboratory data possibly due to different inclusion criteria, authors' specialty, study design, and racial biases. This prompted us to conduct a large-scale multicenter study with well-experienced physicians of IgG4-RD including rheumatologists, gastroenterologists, pulmonologists, and nephrologists to clarify the baseline clinical and laboratory features of IgG4-RD in a large-scale cohort of 334 IgG4-RD patients.

\section{Methods}

\section{Summary of cohort}

We retrospectively evaluated 334 patients with IgG4-RD who were diagnosed between 1996 and 2015 in Kanazawa University Hospital, Sapporo Medical University Hospital, Nagaoka Red Cross Hospital, University of Toyama Hospital, Shinshu University Hospital, and affiliated hospitals. Data related to patients' baseline clinical features, laboratory findings, imaging tests, pathological tests, and treatments were derived from their medical records.

\section{Inclusion criteria}

To minimize bias regarding the organs affected by IgG4$\mathrm{RD}$, the diagnosis of IgG4-RD was made by specialists of IgG4-RD in each institution, supported by the comprehensive diagnostic criteria (CDC) [12] and/or criteria of each organ including type 1 autoimmune pancreatitis, IgG4-related kidney disease, IgG4-related Mikulicz's disease (IgG4-related sialadenitis and dacryoadenitis), and IgG4-related sclerosing cholangitis [6, 13-15]. The CDC consist of three parts including clinical features showing characteristic diffuse/localized swelling or masses in single or multiple organs, elevation of serum IgG4 concentrations $(\geq 135 \mathrm{mg} / \mathrm{dl})$, and histopathological findings including marked lymphoplasmacytic infiltration, fibrosis, and infiltration of IgG4-positive cells $\left(\mathrm{IgG}^{+} / \mathrm{IgG}^{+}>40 \%\right.$ and $>10 \mathrm{IgG}^{+}$plasma cells/HPF). Since the cutoff number of IgG4-positive plasma cells of the CDC is lower than those of the Consensus statement on the pathology of IgG4-RD [16], we made the diagnosis of IgG4-RD referring to the Consensus statement on the pathology by specialists who have treated many IgG4-RD patients and paid particular attention to any clinicopathologic correlations. The histopathological importance of tissue infiltrating IgG4-positive plasma cells (IgG4 $\left.{ }^{+} \mathrm{PC}\right)$ in the diagnosis of AIP was reported in 2002, and was also confirmed in the diagnosis of Mikulicz disease (MD) in 2005, which is currently known as IgG4-related dacryoadenitis and sialadenitis. Therefore, after 2005, almost all patients in our study were diagnosed as having IgG4-RD with reference to the immunohistochemical findings of IgG4 staining, clinical and imaging features, and/or serum IgG4 levels. In contrast, 30 patients who were diagnosed as having AIP or MD before 2005 were also included in our study. Of these, four patients were diagnosed with AIP before 2002 . Reevaluation of histopathological samples was performed in all four of these patients, and the diagnosis of IgG4-RD was confirmed. Similarly, 26 patients were diagnosed with IgG4-related dacryoadenitis and sialadenitis before 2005. Fifteen of these 26 patients were reevaluated histopathologically and the final diagnosis was confirmed. In the remaining 11 patients, eight had bilateral lachrymal and submandibular gland swelling with elevated serum IgG4 levels, with this combination highly specific for the diagnosis of IgG4-RD. The other three patients had only one set of bilateral lacrimal or bilateral submandibular gland swelling, and all of them had an elevated serum IgG4 level. These three patients were diagnosed with IgG4-RD with reference to the clinical and imaging features and elevation of serum IgG4 levels.

The numbers of patients with definite, probable, and possible IgG4-RD according to $\mathrm{CDC}$ and/or organspecific criteria were 280 (83.8\%), 49 (14.7\%), and 4 (1.2\%), respectively. One patient was diagnosed clinically with IgG4-RD (Table 1).

\section{Clinical features}

We retrospectively analyzed the medical records of all patients included in this study. We noted the serum levels of IgG, IgG4, IgE, C3, C4, CH50, and C-reactive protein (CRP), the affected organs, the diagnostic imaging tests including computed tomography (CT), magnetic resonance imaging (MRI), gallium (Ga) scintigraphy, and positron emission tomography (PET), and the prevalence of biopsy of the affected organs. Serum IgG4 levels were measured using the nephelometric assay in all patients in this study. In addition, as a new serological marker, we defined non-IgG4 IgG as total IgG minus IgG4. Non-IgG4 IgG substitutes for the sum of IgG1, IgG2, and IgG3, and an increase of non-IgG4 
Table 1 Baseline clinical features of patients with immunoglobulin G4-related disease

\begin{tabular}{|c|c|}
\hline Characteristic & Value \\
\hline $\begin{array}{l}\text { Mean age at diagnosis (years), } \\
\text { mean } \pm S D \text { (range) }\end{array}$ & $63.8 \pm 11.5(25-91)$ \\
\hline Sex $(n)$, male/female & $205(61.4 \%) / 129(38.6 \%)$ \\
\hline Mean follow-up period (years) & $4.2 \pm 3.3$ \\
\hline Diabetes mellitus, $n$ (\%) & $110 / 321(34.3)$ \\
\hline Autoimmune pancreatitis (-) & $71 / 237(30.0)$ \\
\hline Autoimmune pancreatitis (+) & $39 / 84(46.2)$ \\
\hline \multicolumn{2}{|l|}{ Affected organs, $n(\%)$} \\
\hline Salivary glands & $242(72.7)$ \\
\hline Lacrimal glands & $190(57.1)$ \\
\hline Lymph nodes & $188(56.5)$ \\
\hline Pancreas & $85(25.5)$ \\
\hline Retroperitoneum/periaorta & $83(24.9)$ \\
\hline Kidney & $79(23.7)$ \\
\hline Lung & $78(23.4)$ \\
\hline Prostate & $32(9.6)$ \\
\hline Bile duct & $18(5.4)$ \\
\hline Skin & $5(1.5)$ \\
\hline Thyroid glands & $3(0.9)$ \\
\hline Mean number (range) of affected organs & $3.2(1-11)$ \\
\hline \multicolumn{2}{|l|}{ Number (\%) of affected organs } \\
\hline 1 & $38(11.4)$ \\
\hline 2 & $86(25.7)$ \\
\hline 3 & $84(25.1)$ \\
\hline 4 & $75(22.5)$ \\
\hline 5 & $25(7.5)$ \\
\hline 6 & $18(5.4)$ \\
\hline 7 & $3(0.9)$ \\
\hline 8 & $2(0.6)$ \\
\hline 9 & $2(0.6)$ \\
\hline 10 & $0(0.0)$ \\
\hline 11 & $1(0.3)$ \\
\hline
\end{tabular}

$S D$ standard deviation

IgG seems to mean that the serum level of IgGs which can activate the complement pathway is increased. We also evaluated the prevalence of corticosteroid therapy, average initial and maintenance dose of corticosteroid, effectiveness of corticosteroid therapy, and recurrence rate. The improvement of the affected organs was decided as the changes in symptomatic, radiologic, serologic, or histologic features. In IgG4-related kidney disease (IgG4-RKD), improvement of renal function was also considered. The definition of recurrence was the reappearance or worsening of symptomatic, radiologic, serologic, or histologic features of IgG4-RD. In IgG4-RKD, a rapid increase in the serum level of creatinine, after careful exclusion of other renal diseases, was also considered as recurrence. Reelevation of the serum levels of IgG or IgG4 alone was not regarded as recurrence. Furthermore, we determined the prevalence of diabetes mellitus (DM) at diagnosis of IgG4-RD and malignancy both before and after diagnosis of IgG4-RD.

\section{Statistical analysis}

Differences between groups were assessed by chi-square test for categorical variables, and by Mann-Whitney $U$ test for linear variables. The analysis of the organs associated with hypocomplementemia was performed using logistic regression analysis, adjusted for age, sex, and presence/ absence of DM. Correlation analysis was performed to confirm the relationship between hypocomplementemia and serum levels of IgG4 or non-IgG4 IgG. Explanatory factor analysis followed by Varimax rotation was used to examine the structure of organ coinvolvement. All statistical tests were performed using SPSS software (version 22). Significant differences were defined as $p<0.05$.

\section{Results \\ Patient demographics}

Two hundred and five patients were male, and 129 were female (male 61.4\%). The mean and median ages at diagnosis were $63.8 \pm 11.5$ and 65 years (range 25-91). All patients were Japanese. The average follow-up period was 4.2 years. The prevalence of diabetes mellitus (DM) was $34.4 \%$ and was significantly higher in those with rather than without AIP $(46.2 \%$ vs $30.0 \%, p=0.005)$ (Table 1$)$. Sixty-seven malignancies appeared in 57 of 334 patients (17.1\%) with IgG4-RD (Table 2). Two and six patients had three and two malignancies, respectively. The types of malignancy are presented in Additional file 1: Table S1. Lung cancer was the most frequent malignancy in patients with IgG4-RD, followed by gastric cancer, colon cancer, and prostate cancer. Lung, colon, prostate, and renal cancers were frequently seen both before and after the diagnosis of IgG4-RD, whereas gastric cancer and malignant lymphoma tended to appear after the diagnosis of IgG4-RD (Table 2).

\section{Affected organs}

The mean number of organs involved was 3.2 (range 111). The most frequently affected organs were the salivary glands (SG) $(72.7 \%)$ followed by the lacrimal glands (LG) (57.1\%), lymph nodes (56.5\%), pancreas (25.5\%), retroperitoneum (RP)/periaorta (24.9\%), kidney (23.7\%), and lung (23.4\%). Single organ involvement was seen in 38 of 334 patients (11.4\%) (Table 1). After excluding lymph nodes, the ratio of single organ involvement was $18.9 \%$. The other affected organs with a prevalence of more than $1 \%$ were the prostate $(9.6 \%)$, bile duct $(5.4 \%)$, and skin (1.5\%). The RP/periaorta, lung, and kidney were more frequently affected in males than in females, and 
Table 2 Type of malignancies

\begin{tabular}{|c|c|c|}
\hline Type of malignancy & $\begin{array}{l}\text { Before diagnosis } \\
\text { of lgG4-RD }\end{array}$ & $\begin{array}{l}\text { After diagnosis } \\
\text { of IgG4-RD }\end{array}$ \\
\hline Total & 30 & 36 \\
\hline $\begin{array}{l}\text { Time before or after diagnosis of } \\
\text { lgG4-RD (years) }\end{array}$ & 6.1 & 2.7 \\
\hline Lung cancer & 6 & 6 \\
\hline Colon cancer & 4 & 5 \\
\hline Renal cancer & 4 & 1 \\
\hline Prostate cancer & 3 & 4 \\
\hline Gastric cancer & 2 & 5 \\
\hline Breast cancer & 2 & 0 \\
\hline Malignant lymphoma & 2 & 5 \\
\hline Thyroid cancer & 2 & 2 \\
\hline Bladder cancer & 2 & 1 \\
\hline Cancer of the ovary & 1 & 0 \\
\hline Uterine body cancer & 1 & 0 \\
\hline Urothelial cancer & 1 & 0 \\
\hline Pancreatic cancer & 0 & 1 \\
\hline Cervical cancer & 0 & 1 \\
\hline Leukemia & 0 & 1 \\
\hline Tongue cancer & 0 & 1 \\
\hline Cancer of the throat & 0 & 1 \\
\hline Adenoma sebaceum & 0 & 1 \\
\hline Gastrointestinal stromal tumor & 0 & 1 \\
\hline
\end{tabular}

IgG4-RD immunoglobulin G4-related disease

the reverse for the LG (see Additional file 1: Table S1). The factor analysis identified three factors, explaining $21.7 \%$ of the total variance: factor 1 explained $8.1 \%$, affecting LG, SG, and lymph node involvement; factor 2 explained 7.0\%, affecting RP/periaorta involvement; and factor 3 explained 6.6\%, and affected kidney and lung involvement. These results indicated that organ involvement occurs rather randomly, although there might be some tendency of coinvolvement in the three different organ categories (see Additional file 2: Table S2).

\section{Laboratory data}

Mean serum levels of IgG and IgG4 were $2403 \pm 1204$ and $755 \pm 642 \mathrm{mg} / \mathrm{dl}$, respectively. Elevation of serum levels of IgG4 defined as IgG4 $\geq 135 \mathrm{mg} / \mathrm{dl}$ were seen in 318 of 333 patients (95.5\%). Mean serum level of IgE was $611 \pm 1198 \mathrm{IU} / \mathrm{ml}$, and elevated serum levels defined as $\mathrm{IgE} \geq 250 \mathrm{IU} / \mathrm{ml}$ were seen in 158 of 309 patients (51.1\%). Elevation of serum levels of CRP defined as CRP $\geq 0.3 \mathrm{mg} / \mathrm{dl}$ was seen in 90 of 328 patients (27.4\%). Mean and median serum levels of CRP were 0.42 and $0.10 \mathrm{mg} / \mathrm{dl}$, respectively. In $79.9 \%$ and $90.2 \%$ of the patients, the serum level of CRP was less than 0.5 and $1.0 \mathrm{mg} / \mathrm{dl}$, respectively (Table 3 ).
Table 3 Laboratory findings

\begin{tabular}{|c|c|}
\hline Test item & Value \\
\hline $\operatorname{lgG}(\mathrm{mg} / \mathrm{dl})$ & $2403 \pm 1204$ \\
\hline $\operatorname{lgG} 4(\mathrm{mg} / \mathrm{dl})$ & $755 \pm 642$ \\
\hline Elevation of IgG4 ( $\geq 135)$ & $318 / 333(95.5 \%)$ \\
\hline $\operatorname{lgE}(\mathrm{IU} / \mathrm{ml})$ & $611 \pm 1198$ \\
\hline Elevation of IgE ( $(2250)$ & 158/309 (51.1) \\
\hline CRP (mg/dl) & $0.42 \pm 0.94$ \\
\hline CRP $(\mathrm{mg} / \mathrm{dl})$, median & 0.10 \\
\hline $\mathrm{CRP}<0.5 \mathrm{mg} / \mathrm{dl}$ & $262 / 328(79.9)$ \\
\hline $\mathrm{CRP}<1.0 \mathrm{mg} / \mathrm{dl}$, & $296 / 328(90.2)$ \\
\hline Elevation of CRP ( $\geq 0.3$ ) & $90 / 328(27.4)$ \\
\hline C3 (mg/dl) & $94.6 \pm 31.5$ \\
\hline$C 3<86$ & 103/297 (34.7) \\
\hline $\mathrm{C} 4$ (mg/dl) & $21.2 \pm 23$ \\
\hline$C 4<17$ & 100/297 (33.7) \\
\hline $\mathrm{CH} 50(\mathrm{U} / \mathrm{ml})$ & $41.1 \pm 15.5$ \\
\hline $\mathrm{CH} 50<25$ & 46/292 (15.8) \\
\hline
\end{tabular}

Data presented as mean \pm standard deviation or number/total (\%), unless indicated otherwise

CRP C-reactive protein, lgE immunoglobulin E, lgG4 immunoglobulin G4

Patients with low C3 and C4 defined as C3 $<86 \mathrm{mg} / \mathrm{dl}$ and $\mathrm{C} 4<17 \mathrm{mg} / \mathrm{dl}$ were seen in $34.7 \%$ and $33.7 \%$, respectively. The frequency of low $\mathrm{C} 3$ in patients with kidney lesion was significantly higher than in those without $(55.4 \%$ vs $27.8 \%, p<0.000)$ (Table 4$)$. The frequency of low C3 in patients with pancreas or lung involvement was also significantly higher than in those without (pancreas $46.8 \%$ vs $30.3 \%, p=0.009$; lung $47.2 \%$ vs $30.7 \%, p$ $=0.015$ ) (Table 4). We performed logistic regression analysis to explore the impact of organ involvement on hypocomplementemia, as well as that on severe hypocomplementemia. Kidney, lung, or pancreas involvement was independently related to hypocomplementemia, with odds ratios of 2.60 (95\% CI 1.47-4.59, $p=0.001$ ), 1.84 (95\% CI 1.04-3.26), and 1.83 (95\% CI 1.04-3.21), respectively (Table 5). Patients with severe hypocomplementemia defined as $\mathrm{C} 3<50 \mathrm{mg} / \mathrm{dl}$ were seen in 30 of

Table 4 Frequency of hypocomplementemia

\begin{tabular}{llll}
\hline & \multicolumn{3}{l}{ Frequency of hypocomplementemia } \\
\hline Affected organ & Present & Absent & $p$ \\
Kidney & $41 / 74(55.4 \%)$ & $62 / 223(27.8 \%)$ & $<0.000$ \\
Pancreas & $37 / 79(46.8 \%)$ & $66 / 218(30.3 \%)$ & 0.009 \\
Lung & $34 / 72(47.2 \%)$ & $69 / 225(30.7 \%)$ & 0.015 \\
Lacrimal glands & $52 / 162(32.1 \%)$ & $51 / 135(37.8 \%)$ & 0.329 \\
Retroperitoneum/periaorta & $30 / 76(39.5 \%)$ & $73 / 221(33.0 \%)$ & 0.330 \\
Salivary glands & $76 / 214(35.5)$ & $27 / 83(32.5 \%)$ & 0.685 \\
Lymph nodes & $61 / 171(35.7 \%)$ & $37 / 111(33.3 \%)$ & 0.703 \\
\hline
\end{tabular}

Data presented as number/total (\%) 
Table 5 Factors related to hypocomplementemia

\begin{tabular}{|c|c|c|c|}
\hline & Odds ratio & $95 \% \mathrm{Cl}$ & $p$ \\
\hline \multicolumn{4}{|c|}{ Hypocomplementemia $(C 3<86)$} \\
\hline Kidney & 2.602 & $1.474-4.593$ & 0.001 \\
\hline Lung & 1.844 & $1.044-3.256$ & 0.035 \\
\hline Pancreas & 1.830 & $1.044-3.209$ & 0.035 \\
\hline \multicolumn{4}{|c|}{ Severe hypocomplementemia $(C 3<50)$} \\
\hline Kidney & 6.091 & $2.607-14.229$ & $<0.001$ \\
\hline Lung & 2.474 & $1.054-5.502$ & 0.037 \\
\hline Diabetes mellitus & 2.376 & $1.012-5.502$ & 0.043 \\
\hline
\end{tabular}

297 patients (10.1\%). In a logistic regression analysis, kidney or lung involvement, as well as DM, but not pancreas involvement, were strongly related to severely low C3, with odds ratios of 6.09 (95\% CI 2.61-14.2), 2.47 (95\% CI 1.05-5.50), and 2.38 (95\% CI 1.01-5.50), respectively (Table 5). Next, we analyzed whether serum levels of IgG4 or non-IgG4 IgG, which is calculated as total IgG minus IgG4, were correlated with hypocomplementemia. In an analysis of all patients, the serum level of C3 was significantly inversely correlated with both serum levels of IgG4 and non-IgG4 IgG (Pearson's product-moment correlation coefficient $-0.298, p<0.001$ and $-0.352, p<0.001$, respectively) (Fig. 1a, b), which indicated that low serum C3 level tended to occur in patients with high serum levels of both IgG4 and non-IgG4 IgG. On the other hand, in patients with kidney lesion, the serum C3 level was significantly inversely correlated with only serum levels of non-IgG4 IgG, which indicated that IgG4 may not affect the deposition of C3 in kidney tissue (Fig. 1c, d).

\section{Diagnostic tools}

CT was performed in $99.4 \%$ of patients and contrastenhanced CT in 93.1\%. MRI, PET, and Ga scintigraphy were also performed in $30.2 \%, 45.5 \%$, and $35.1 \%$ of patients, respectively, to determine the extent of systemic involvement. According to the histopathological evaluations, the most frequently biopsied organ were the SG (49.0\%), followed by the kidney (37.5\%), LG (35.1\%), lung $(33.8 \%)$, pancreas $(12.8 \%)$, and $\mathrm{RP} /$ periaorta $(1.2 \%)$ (Fig. 2). These data indicated that the major SG were the most easily accessible organ in patients with IgG4-RD. In contrast, only a single patient underwent $\mathrm{RP} /$ periaorta biopsy because these deeper structures are much more difficult to access.

\section{Treatment}

Corticosteroid therapy was administered to 245 of 314 patients (78.0\%). The mean initial and maintenance dose of prednisolone was 30.5 and $4.1 \mathrm{mg} /$ day, respectively. Corticosteroid was effective in all patients. Recurrence was noted in 67 of 314 patients (21.3\%), in whom 23 of

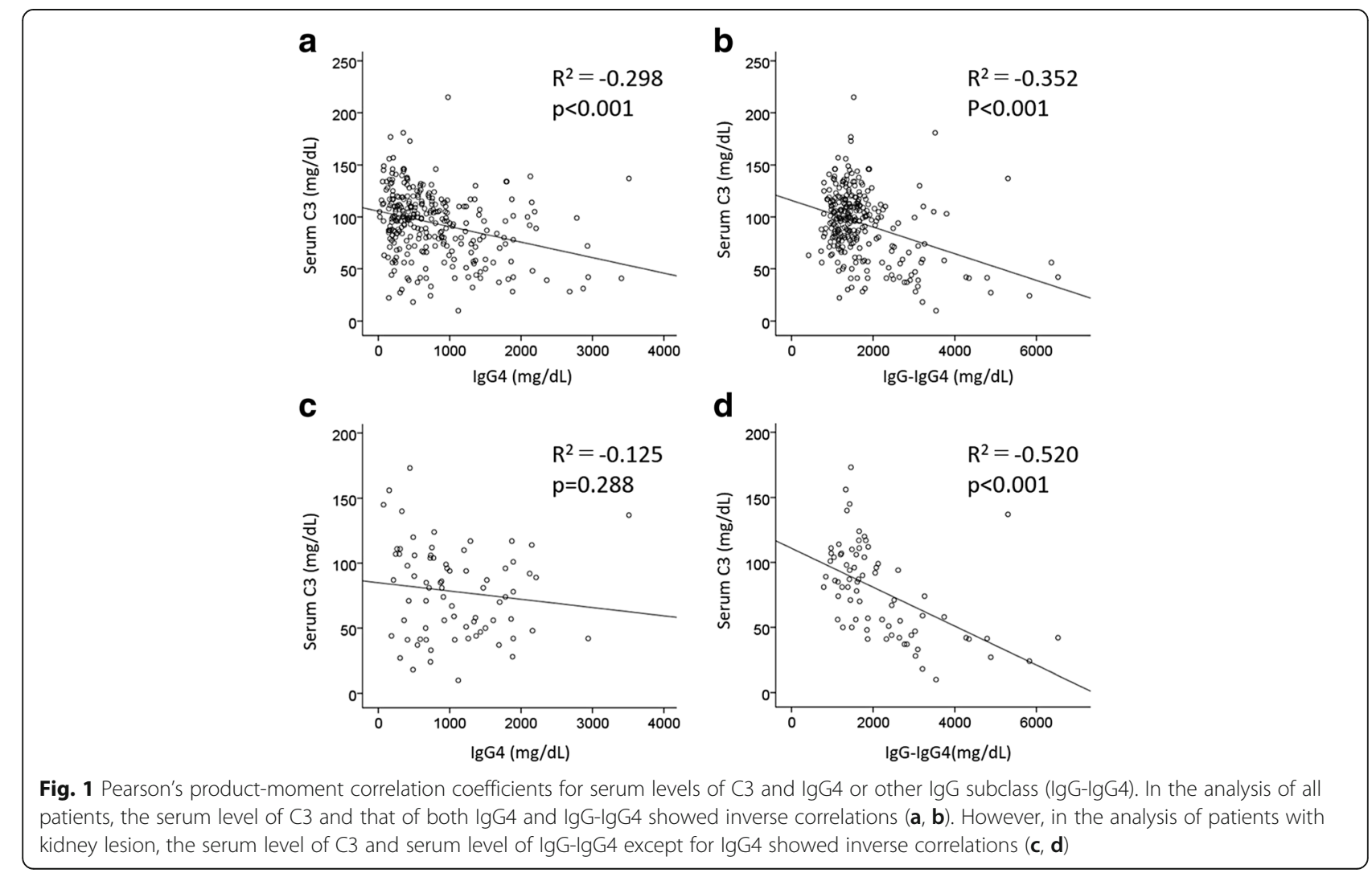




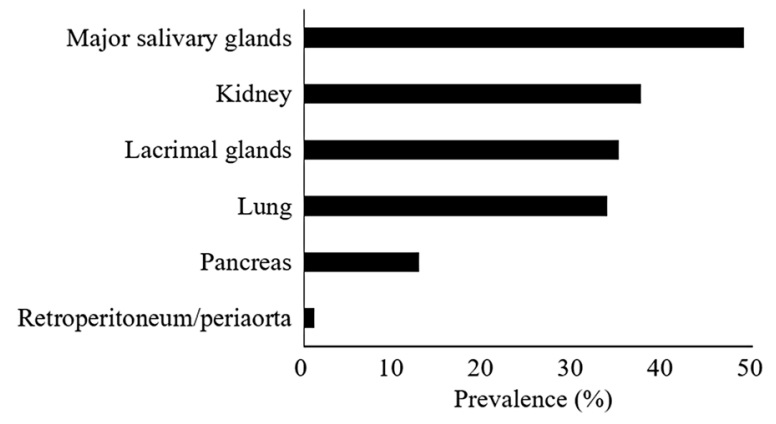

Fig. 2 Type of affected organ biopsied. Major salivary glands were the most frequently biopsied organ, whereas the pancreas and retroperitoneum/periaorta were seldom biopsied

$67(34.3 \%)$ were not receiving corticosteroid at the time of recurrence. The average dose of corticosteroid at first recurrence was $7.1 \mathrm{mg} / \mathrm{dl}$.

\section{Discussion}

To the best of our knowledge, this is the largest cohort study thus far to collect IgG4-RD patients and analyze their clinical features. The salient findings of our cohort study can be summarized as follows. First, elevated serum levels of IgG4 were seen in more than 95\% of patients with IgG4-RD. Second, the median serum CRP level was $0.1 \mathrm{mg} / \mathrm{dl}$, and the serum CRP level was less than $1 \mathrm{mg} / \mathrm{dl}$ in $90.2 \%$ of the patients, meaning that serum levels of CRP are within the normal range in most IgG4-RD patients in Japan. Third, low C3 was seen in $34.7 \%$ of patients, and its frequency differed depending on the organs affected. The affected organs associated with low serum levels of $\mathrm{C} 3$ were the kidney, lung, and pancreas. Serum levels of C3 and non-IgG4 IgG calculated as total IgG minus IgG4 showed inverse correlations in patients with kidney lesion, while serum IgG4 levels did not correlate with serum C3 levels. Fourth, the most frequently affected organs were the salivary glands, lacrimal glands, pancreas, retroperitoneum/periaorta, kidneys, and lungs.

Major differences have been noted in the prevalence of affected organs in previously published studies [9-11]. Wallace et al. [9] analyzed IgG4-RD patients all of whom were subjected to pathological analysis. In their study, the prevalence of organs that were difficult to access, such as the pancreas, was relatively low and $19.2 \%$ of patients had type I AIP. Inoue at al. [10] used a radiology database to detect IgG4-RD patients who were pathologically diagnosed with IgG4-RD and/or fulfilled the diagnostic criteria of AIP. This method picked up AIP patients without pathological analysis and showed that $61 \%$ of patients had pancreas lesions. However, the prevalence of retroperitoneal and/or periaortal lesions was very low (both $4 \%$ ) in both analyses, and the issue of how to best diagnose involvement of organs which are difficult to access remained unresolved. In our study, patients with retroperitoneal and/or periaortal lesions were diagnosed as having IgG4-RD without biopsy through clinicoimaging correlations obtainable in daily medical practice. We found six organs to be the most frequently affected: salivary glands, lacrimal glands, pancreas, retroperitoneum/periaorta, kidney, and lung, with the last four organs having almost the same prevalence (around $25 \%)$. The major affected organs could be classified into the following three subgroups using Varimax rotation: group 1, lacrimal glands, salivary glands, and lymph node; group 2, retroperitoneum/periaorta; and group 3, kidney and lung (see Additional file 2). Inoue et al. [10] reported that male and female patients showed a different distribution of organ manifestations. They noted that dacryoadenitis and sialadenitis developed more commonly in females (female-to-male ratio 41:18 and 57:29 respectively), while periaortitis was significantly more common in males than females (female-to-male ratio $2: 25)$. Thus, our results of factor analysis are consistent with their results. Although we are not yet able to clarify the factors particular to each subgroup, recognition of these subgroups provides a good opportunity to speculate on the different pathogenetic mechanisms at work in each of them.

In this study, the serum IgG4 level was elevated in $95 \%$ of patients. Inoue et al. [10] analyzed 235 patients and found elevated serum IgG4 levels in $88 \%$ of them. Similarly, a large Chinese cohort study [11] showed that $97.5 \%$ of patients had serum IgG4 elevation. The results of these studies suggest that most patients with IgG4$\mathrm{RD}$ in Asian countries have increased serum IgG4 levels (Table 6). In contrast, Wallace et al. [9] analyzed 125 patients with IgG4-RD and showed that only 51\% of them had elevated serum IgG4 concentrations. In this study, $76 \%$ were Caucasian and only $8.8 \%$ were Asian. Therefore, the difference in the proportion of patients with elevated serum IgG4 in these studies may be due at least in part to racial differences. Another important factor possibly influencing the frequency of patients with high serum IgG4 is the proportion of patients with single organ involvement in each study. In the study by Wallace et al. [9] $38 \%$ of patients had single organ involvement, in contrast to $11 \%$ in our study. Similarly, only $4.2 \%$ had one organ involved in Lin et al.'s study [11] (Table 6). Since patients with single organ involvement tend to have low serum IgG4 levels, the percentage of such patients is thought to influence the frequency of high serum IgG4 patients.

In our study, elevation of the serum level of CRP was seen only in $27.4 \%$ of patients. The median serum level of CRP was $0.1 \mathrm{mg} / \mathrm{dl}$, and values less than 0.5 and $1.0 \mathrm{mg} / \mathrm{dl}$ were present in about $80 \%$ and $90 \%$ of IgG4- 
Table 6 Comparison of laboratory findings and percentage of cases with single organ involvement between the present study and previous reports

\begin{tabular}{|c|c|c|c|c|c|c|c|}
\hline & Present study & Inoue et al. [10] & Lin et al. [11] & $\begin{array}{l}\text { Campochiaro } \\
\text { et al. [22] }\end{array}$ & Ebbo et al. [23] & $\begin{array}{l}\text { Fernández-Codina } \\
\text { et al. [24] }\end{array}$ & $\begin{array}{l}\text { Wallace } \\
\text { et al. [9] }\end{array}$ \\
\hline Reported year & & 2015 & 2015 & 2015 & 2012 & 2015 & 2015 \\
\hline Number of patients & 334 & 235 & 118 & 41 & 25 & 55 & 125 \\
\hline Country & Japan & Japan & China & Italy & France & Spain & USA \\
\hline Mean serum lgG level (mg/dl) & 2403 & NA & 2300 & NA & 2550 & NA & $1573^{\mathrm{d}}, 1130^{\mathrm{e}}$ \\
\hline Mean serum lgG4 level (mg/dl) & 755 & $470^{a}$ & 1522 & $284^{\mathrm{a}}$ & 720 & $223^{\mathrm{a}, \mathrm{b}}, 93^{\mathrm{a}, \mathrm{c}}$ & $379^{d}, 51^{e}$ \\
\hline Elevation of IgG4 (\%) & 95.5 & 88 & 97.5 & 73 & 100 & NA & 51.4 \\
\hline Median serum CRP level (mg/dl) & 0.10 & NA & NA & 0.8 & NA & NA & $0.55^{\mathrm{d}}, 0.65^{\mathrm{e}}$ \\
\hline Mean serum CRP level (mg/dl) & 0.42 & NA & 0.66 & NA & 3.09 & NA & NA \\
\hline CRP $<0.5$ mg/dl (\%) & 79.9 & NA & NA & NA & NA & NA & NA \\
\hline CRP < 1.0 mg/dl (\%) & 90.2 & NA & NA & NA & 46 & NA & NA \\
\hline Elevation of CRP (\%) & 27.4 & NA & 44.1 & 63 & 56 & NA & $67^{\mathrm{d}, \mathrm{f}}, 46^{\mathrm{e}, \mathrm{f}}$ \\
\hline Single organ involvement (\%) & 11.4 & 42 & 4.2 & 58.5 & 12.0 & 52.7 & 38 \\
\hline
\end{tabular}

CRP C-reactive protein, IgG4 immunoglobulin G4, IgG4-RD immunoglobulin G4-related disease, NA not applicable

${ }^{\text {a Median value }}$

'Highly suggestive lgG4-RD

'Probable lgG4-RD

dPatients with elevated serum IgG4 concentration

ePatients with normal serum IgG4 concentration

'Erythrocyte sedimentation rate and/or CRP

RD patients, respectively. These data indicate that serum levels of CRP in most patients with IgG4-RD are normal or low. Elevated serum IgG4 and pathological features mimicking those of IgG4-RD have been noted in other diseases such as multicentric Castleman disease (MCD) [17] and anti-neutrophil cytoplasmic antibody (ANCA)associated vasculitis, and in particular granulomatosis with polyangiitis (GPA) [18] and eosinophilic granulomatosis with polyangiitis (EGPA) [19, 20]. Important for the differential diagnosis is the fact that serum CRP levels are generally elevated in these diseases [21], making the serum CRP level an important serological marker in differentiating IgG4-RD in Japanese patients. However, the fact that $44-67 \%$ of patients had elevated CRP levels in cohort studies from western countries $[9,22-24]$ indicates that interpretation of the serum CRP level may have to take into account racial and possibly other differences in the subjects and populations studied (Table 6).

Hypocomplementemia is an important serological feature of IgG4-RD, in particular IgG4-RKD $[6,25,26]$. More than $50 \%$ of IgG4-RKD patients have hypocomplementemia, while the prevalence of hypocomplementemia is about $30 \%$ in all IgG4-RD. This has led to speculation about the reasons underlying the strong association noted between hypocomplementemia and IgG4-related tubulointerstitial nephritis (TIN). In the present study, we noted low C3 and C4 levels in 35\% and 34\%, respectively, of IgG4-RD patients. Furthermore, hypocomplementemia in patients with kidney lesion was significantly more frequent than in those without, supporting previous reports.

Interestingly, serum complement levels rapidly normalize after successful corticosteroid therapy in parallel with the reduction of the size of mass or enlarged lesions [26]. Moreover, a renewed decrease in serum complement levels has been observed during the clinical course of relapse in patients with IgG4-related TIN [27], highlighting hypocomplementemia as a convenient biomarker of disease activity. However, in general, IgG4 has been thought not to bind to $\mathrm{C} 1 \mathrm{q}$, which is related to its inability to activate the classical complement pathway. On the other hand, one report showed that IgG4 molecules derived from patients with IgG4-RD and hypocomplementemia can activate complement through C1q-binding [28], although additional studies to confirm this are necessary. Previous histopathological immunofluorescent analyses showed that not only IgG4 but also IgG1 is deposited in the tubular basement membrane of the kidney. This suggests that IgG1 also has a pathogenetic role in IgG4-RKD. Using a mouse model of AIP, Shiokawa et al. [29] showed that more destructive injury in the pancreas was induced by injecting the patient with IgG1 rather than IgG4. In the present study, we found that serum levels of non-IgG4 IgG were significantly inversely correlated with serum C3 levels in patients with kidney lesion, while IgG4 itself has only a weak correlation with serum C3 levels. Our finding seems to be compatible with Shiokawa et al.'s result. To the best of our knowledge, the present study is the first report to show that non-IgG4 
IgG subclasses exert a more significant influence on hypocomplementemia, probably through activation of complement in patients with kidney lesions, than IgG4 subclass. Thus, IgG subclasses other than IgG4 should also be focused on as pathogenetic immunoglobulins.

The rate of malignancies in this study (17.1\%) was higher than those in other Japanese cohort studies $(6.4 \%$ [10], 10.4\% [30], 13.9\% [31], 12.3\% [32]) but lower than that of the study reported by Asano et al. (21.5\% [33]). Of these five studies, three confirmed an increased risk of malignancy in IgG4-RD using the standardized incidence ratio (SIR), while the other two did not. Since we did not calculate the SIR because of the limitation of our study design, we could not easily compare the frequency of malignancy with that in other Japanese studies. The discordant results found in Japanese studies are likely attributable to methodological issues related to the study design of individual studies. Hirano et al. [32] excluded patients in whom malignancy was diagnosed $\leq 6$ months before or after the onset of IgG4-RD to avoid selection bias, whereas other studies did not. Thus, careful evaluation is needed to conclude that the rate of malignancies in IgG4-RD is significantly higher than that of controls.

The recurrence rate of our study was lower than those of western countries. Reports from Spain [24] and Italy [22] described the recurrence rate to be $38.5 \%$ and $46 \%$, respectively. On the other hand, Inoue et al. [10] noted the recurrence rate in Japanese patients to be $24 \%$, almost the same as in our study. A recent meta-analysis [34] also showed that the recurrence rates in Americans and Europeans were higher than those in Asians. The difference in the recurrence rates between Japanese and Caucasians may be attributable to differences in the therapeutic strategy, especially the use or nonuse of corticosteroid maintenance therapy, race, and various other patient background features. However, we did not analyze the relationship between the treatment strategy for IgG4-RD such as the duration and tapering pace of corticosteroid and immunosuppressant administration and the recurrence rate. This important issue will require further studies for clarification.

Our study has several limitations. First, biopsies of the affected organs were not performed for $18.9 \%$ of the patients because the affected organs were not easily accessible. Second, methodological differences in the measurement of laboratory parameters such as serum complement levels among different periods and different institutions is a weak, but unavoidable, aspect of our retrospective cohort study. However, the effect of the difference of the method employed among different periods and different institutions on complement levels is limited and the influence would seem to be quite small. On the other hand, the serum IgG4 level was measured using nephelometry in all institutions. Therefore, we think no methodological difference existed. Third, we used the serum levels of non-IgG4 IgG instead of those of $\operatorname{IgG1}$ IgG2, or $\operatorname{IgG} 3$, because we do not measure serum levels of IgG1, IgG2, and IgG3 in daily clinical practice.

It remains difficult to clarify the frequency of individual organs affected by IgG4-RD due to various biases related to the criteria employed for diagnosis and differences in the distribution of the specialties of clinicians making the diagnosis of IgG4-RD. In this study, we believe that we were better able to elucidate the baseline clinical features of IgG4-RD by having specialists of IgG4-RD from a variety of fields participate in the evaluations.

Another issue highlighted by this study is the finding of differences in various clinical parameters between our population and western ones. This raises the important question of whether the hitherto proposed international consensus criteria are equally applicable to all populations, and whether the more or less universally recognized clinical spectrum of IgG4-RD is the same in all of them. Further exploration of the reasons underlying any differences may also provide intriguing clues to the underlying nature of IgG4-RD and be of use in devising more accurate diagnostic criteria. To best characterize IgG4-RD and assess the universality of various criteria for diagnosis, future studies, including international comparative ones, will be needed with larger numbers of IgG4-RD patients diagnosed according to various criteria.

\section{Conclusions}

In Japanese individuals, various clinical parameters in IgG4-RD including serum IgG4 and CRP levels appear to differ in some interesting ways from those hitherto reported in western populations. Additional studies, especially international comparative ones, will be needed to elucidate the extent and significance of these differences between populations. Attention will also have to be paid to whether such differences require consideration when devising diagnostic criteria that are meant to be applied internationally.

\section{Additional files}

Additional file 1: Table S1. Gender differences in affected organs. (DOC $64.5 \mathrm{~kb}$ )

Additional file 2: Table S2. Factor analysis of the affected organs. (DOC $33 \mathrm{~kb}$ )

\section{Abbreviations}

CDC: Comprehensive diagnostic criteria; $C T$ : Computed tomography; DM: Diabetes mellitus; EGPA: Eosinophilic granulomatosis with polyangiitis; Ga: Gallium; IgG4: Immunoglobulin G4; IgG4-RD: Immunoglobulin G4-related disease; IgG4-RKD: Immunoglobulin G4-related kidney disease; LG: Lacrimal glands; MCD: Multicentric Castleman disease; MRI: Magnetic resonance imaging; PET: Positron emission tomography; RP: Retroperitoneum; SG: Salivary glands; TIN: Tubulointerstitial nephritis 


\section{Acknowledgments}

The authors thank Mr John Gelblum and Mr Eric Tusken for their critical reading of the manuscript.

\section{Funding}

This work was supported partially by the Research Program of Intractable Diseases of the Ministry of Health, Labor, and Welfare of Japan, JSPS KAKENHI Grant Number 26461487 and 17 K09999, and a grant-in-aid from Kato Memorial Nambyo Research.

\section{Availability of data and materials}

Not applicable.

\section{Authors' contributions}

KY designed the study, acquired data, contributed to analysis and interpretation of data, and drafted the manuscript. MY acquired data and provided intellectual content of critical importance to the work described. TS acquired data, contributed to analysis and interpretation of data, and provided intellectual content of critical importance to the work described. IM acquired data and provided intellectual content of critical importance to the work described. SM acquired data and provided intellectual content of critical importance to the work described. YF acquired data, contributed to analysis and interpretation of data, and provided intellectual content of critical importance to the work described. SH acquired data and provided intellectual content of critical importance to the work described. HT acquired data and provided intellectual content of critical importance to the work described. HN acquired data and provided intellectual content of critical importance to the work described. SK acquired data and provided intellectual content of critical importance to the work described. MK designed the study, contributed to analysis and interpretation of data, provided intellectual content of critical importance to the work described, and revised the manuscript. All authors read and approved the final manuscript.

\section{Ethics approval and consent to participate}

This study was approved by the ethics committees of Kanazawa University Hospital, Sapporo Medical University Hospital, Nagaoka Red Cross Hospital, University of Toyama Hospital, and Shinshu University Hospital. All data and samples from patients were collected with their informed consent, and the study was conducted in compliance with the Declaration of Helsinki Principles.

\section{Consent for publication}

Not applicable.

\section{Competing interests}

The authors declare that they have no competing interests.

\section{Publisher's Note}

Springer Nature remains neutral with regard to jurisdictional claims in published maps and institutional affiliations.

\section{Author details}

'Division of Rheumatology, Kanazawa University Hospital, 13-1, Takara-machi, Kanazawa, Ishikawa 920-8640, Japan. ${ }^{2}$ Department of Advanced Research in Community Medicine, Kanazawa University Graduate School of Medical Sciences, Kanazawa, Japan. ${ }^{3}$ Department of Rheumatology and Clinical Immunology, Sapporo Medical University School of Medicine, Sapporo, Japan. ${ }^{4}$ Department of Internal Medicine, Nagaoka Red Cross Hospital, Nagaoka, Japan. ${ }^{5}$ Health Administration Center, University of Toyama, Toyama, Japan. ${ }^{6}$ Division of General Medicine, Kanazawa University Hospital, Kanazawa, Japan. 'Department of Internal Medicine, Matsumoto Dental University, Shiojiri, Japan

\section{Received: 9 June 2017 Accepted: 7 November 2017} Published online: 01 December 2017

\section{References}

1. Hamano H, Kawa S, Horiuchi A, Unno H, Furuya N, Akamatsu T, Fukushima M, Nikaido T, Nakayama K, Usuda N, et al. High serum lgG4 concentrations in patients with sclerosing pancreatitis. N Engl J Med. 2001;344(10):732-8.
2. Stone JH, Zen Y, Deshpande V. IgG4-related disease. N Engl J Med. 2012;366(6):539-51.

3. Masaki Y, Dong L, Kurose N, Kitagawa K, Morikawa Y, Yamamoto M, Takahashi H, Shinomura Y, Imai K, Saeki T, et al. Proposal for a new clinical entity, IgG4-positive multiorgan lymphoproliferative syndrome: analysis of 64 cases of IgG4-related disorders. Ann Rheum Dis. 2009;68(8):1310-5.

4. Kamisawa T, Zen Y, Pillai S, Stone JH. IgG4-related disease. Lancet. 2015;385(9976):1460-71.

5. Yamamoto M, Ohara M, Suzuki C, Naishiro Y, Yamamoto H, Takahashi H, Imai K. Elevated lgG4 concentrations in serum of patients with Mikulicz's disease. Scand J Rheumatol. 2004;33(6):432-3.

6. Kawano M, Saeki T, Nakashima H, Nishi S, Yamaguchi Y, Hisano S, Yamanaka $\mathrm{N}$, Inoue D, Yamamoto M, Takahashi H, et al. Proposal for diagnostic criteria for lgG4-related kidney disease. Clin Exp Nephrol. 2011;15(5):615-26.

7. Saeki T, Nishi S, Ito T, Yamazaki H, Miyamura S, Emura I, Imai N, Ueno M, Saito A, Gejyo F. Renal lesions in IgG4-related systemic disease. Intern Med. 2007:46(17):1365-71.

8. Cheuk W, Yuen HK, Chu SY, Chiu EK, Lam LK, Chan JK. Lymphadenopathy of IgG4-related sclerosing disease. Am J Surg Pathol. 2008;32(5):671-81.

9. Wallace ZS, Deshpande V, Mattoo H, Mahajan VS, Kulikova M, Pillai S, Stone JH. IgG4-related disease: clinical and laboratory features in one hundred twenty-five patients. Arthritis Rheumatol. 2015;67(9):2466-75.

10. Inoue D, Yoshida K, Yoneda N, Ozaki K, Matsubara T, Nagai K, Okumura K, Toshima F, Toyama J, Minami T, et al. IgG4-related disease: dataset of 235 consecutive patients. Medicine (Baltimore). 2015;94(15):e680.

11. Lin W, Lu S, Chen H, Wu Q, Fei Y, Li M, Zhang X, Tian X, Zheng W, Leng X, et al. Clinical characteristics of immunoglobulin G4-related disease: a prospective study of 118 Chinese patients. Rheumatology (Oxford). 2015:54(11):1982-90

12. Umehara H, Okazaki K, Masaki Y, Kawano M, Yamamoto M, Saeki T, Matsui S, Yoshino T, Nakamura S, Kawa S, et al. Comprehensive diagnostic criteria for lgG4-related disease (IgG4-RD), 2011. Mod Rheumatol. 2012;22(1):21-30.

13. Shimosegawa T, Chari ST, Frulloni L, Kamisawa T, Kawa S, Mino-Kenudson M Kim MH, Kloppel G, Lerch MM, Lohr M, et al. International consensus diagnostic criteria for autoimmune pancreatitis: guidelines of the International Association of Pancreatology. Pancreas. 2011:40(3):352-8.

14. Masaki Y, Sugai S, Umehara H. IgG4-related diseases including Mikulicz's disease and sclerosing pancreatitis: diagnostic insights. J Rheumatol. 2010:37(7):1380-5.

15. Ohara H, Okazaki K, Tsubouchi H, Inui K, Kawa S, Kamisawa T, Tazuma S, Uchida K, Hirano K, Yoshida H, et al. Clinical diagnostic criteria of lgG4-related sclerosing cholangitis 2012. J Hepatobiliary Pancreat Sci. 2012;19(5):536-42.

16. Deshpande V, Zen Y, Chan JK, Yi EE, Sato Y, Yoshino T, Kloppel G, Heathcote $J G$, Khosroshahi A, Ferry JA, et al. Consensus statement on the pathology of lgG4-related disease. Mod Pathol. 2012;25(9):1181-92.

17. Sato Y, Kojima M, Takata K, Morito T, Mizobuchi K, Tanaka T, Inoue D, Shiomi H, Iwao H, Yoshino T. Multicentric Castleman's disease with abundant IgG4positive cells: a clinical and pathological analysis of six cases. J Clin Pathol. 2010;63(12):1084-9.

18. Chang SY, Keogh KA, Lewis JE, Ryu JH, Cornell LD, Garrity JA, Yi ES. IgG4 positive plasma cells in granulomatosis with polyangiitis (Wegener's): a clinicopathologic and immunohistochemical study on 43 granulomatosis with polyangiitis and 20 control cases. Hum Pathol. 2013;44(11):2432-7.

19. Yamamoto M, Takahashi H, Suzuki C, Tabeya T, Ohara M, Naishiro Y, Yamamoto H, Imai K, Shinomura Y. Analysis of serum IgG subclasses in Churg-Strauss syndrome-the meaning of elevated serum levels of IgG4. Intern Med. 2010;49(14):1365-70.

20. Vaglio A, Strehl JD, Manger B, Maritati F, Alberici F, Beyer C, Rech J, Sinico RA, Bonatti F, Battistelli L, et al. lgG4 immune response in Churg-Strauss syndrome. Ann Rheum Dis. 2012;71(3):390-3.

21. Suresh E. Diagnostic approach to patients with suspected vasculitis. Postgrad Med J. 2006;82(970):483-8.

22. Campochiaro C, Ramirez GA, Bozzolo EP, Lanzillotta M, Berti A, Baldissera E, Dagna L, Praderio L, Scotti R, Tresoldi M, et al. IgG4-related disease in Italy: clinical features and outcomes of a large cohort of patients. Scand I Rheumatol. 2016;45(2):135-45.

23. Ebbo M, Daniel L, Pavic M, Seve P, Hamidou M, Andres E, Burtey S, Chiche L, Serratrice J, Longy-Boursier M, et al. IgG4-related systemic disease: features and treatment response in a French cohort: results of a multicenter registry. Medicine (Baltimore). 2012;91(1):49-56.

24. Fernandez-Codina A, Martinez-Valle F, Pinilla B, Lopez C, DeTorres I, Solans-Laque R, Fraile-Rodriguez G, Casanovas-Martinez A, Lopez-Dupla 
M, Robles-Marhuenda A, et al. IgG4-related disease: results from a multicenter Spanish registry. Medicine (Baltimore). 2015;94(32):e1275.

25. Saeki T, Ito T, Yamazaki H, Imai N, Nishi S. Hypocomplementemia of unknown etiology: an opportunity to find cases of lgG4-positive multiorgan lymphoproliferative syndrome. Rheumatol Int. 2009;30(1):99-103.

26. Mizushima I, Inoue D, Yamamoto M, Yamada K, Saeki T, Ubara Y, Matsui S, Masaki Y, Wada T, Kasashima S, et al. Clinical course after corticosteroid therapy in IgG4-related aortitis/periaortitis and periarteritis: a retrospective multicenter study. Arthritis Res Ther. 2014;16(4):R156.

27. Saeki T, Kawano M, Mizushima I, Yamamoto M, Wada Y, Nakashima H, Homma N, Tsubata Y, Takahashi H, Ito T, et al. The clinical course of patients with IgG4-related kidney disease. Kidney Int. 2013;84(4):826-33.

28. Sugimoto M, Watanabe H, Asano T, Sato S, Takagi T, Kobayashi H, Ohira H. Possible participation of lgG4 in the activation of complement in lgG4-related disease with hypocomplementemia. Mod Rheumatol. 2016;26(2):251-8.

29. Shiokawa M, Kodama Y, Kuriyama K, Yoshimura K, Tomono T, Morita T, Kakiuchi N, Matsumori T, Mima A, Nishikawa Y, et al. Pathogenicity of lgG in patients with IgG4-related disease. Gut. 2016;65(8):1322-32.

30. Yamamoto M, Takahashi H, Tabeya T, Suzuki C, Naishiro Y, Ishigami K, Yajima H, Shimizu Y, Obara M, Yamamoto H, et al. Risk of malignancies in IgG4-related disease. Mod Rheumatol. 2012;22(3):414-8.

31. Shiokawa M, Kodama Y, Yoshimura K, Kawanami C, Mimura J, Yamashita Y, Asada M, Kikuyama M, Okabe $Y$, Inokuma T, et al. Risk of cancer in patients with autoimmune pancreatitis. Am J Gastroenterol. 2013;108(4):610-7.

32. Hirano K, Tada M, Sasahira N, Isayama H, Mizuno S, Takagi K, Watanabe T, Saito T, Kawahata S, Uchino R, et al. Incidence of malignancies in patients with IgG4-related disease. Intern Med. 2014;53(3):171-6.

33. Asano J, Watanabe T, Oguchi T, Kanai K, Maruyama M, Ito T, Muraki T, Hamano $\mathrm{H}$, Arakura N, Matsumoto A, et al. Association between immunoglobulin G4related disease and malignancy within 12 years after diagnosis: an analysis after longterm followup. J Rheumatol. 2015;42(11):2135-42.

34. Brito-Zeron P, Kostov B, Bosch X, Acar-Denizli N, Ramos-Casals M, Stone JH. Therapeutic approach to lgG4-related disease: a systematic review. Medicine (Baltimore). 2016;95(26):e4002.

\section{Submit your next manuscript to BioMed Central and we will help you at every step:}

- We accept pre-submission inquiries

- Our selector tool helps you to find the most relevant journal

- We provide round the clock customer support

- Convenient online submission

- Thorough peer review

- Inclusion in PubMed and all major indexing services

- Maximum visibility for your research

Submit your manuscript at www.biomedcentral.com/submit 